\title{
Comparison of monoclonal gammopathy of undetermined significance-associated neuropathy and chronic inflammatory demyelinating polyneuropathy patients
}

\author{
Nuha M. Alkhawajah • Samantha K. Dunnigan • \\ Vera Bril
}

Received: 17 December 2013/Revised: 16 April 2014/ Accepted: 17 April 2014/Published online: 7 May 2014

(C) The Author(s) 2014. This article is published with open access at Springerlink.com

\begin{abstract}
Objectives There are varying reports on whether monoclonal gammopathy of undetermined significance-associated neuropathy (MGUSN) patients are distinguishable from those with chronic inflammatory demyelinating polyneuropathy (CIDP) and whether specific MGUSN subclasses are associated with specific clinical phenotypes.

Methods We performed a retrospective chart review of MGUSN $(n=56)$ and CIDP $(n=67)$ patients. Data extracted included: demographics, neurological examination, and nerve conduction studies (NCS) at baseline and last visit. Clinical status was rated as $0=$ worse, $1=$ unchanged, $2=$ stabilized after a declining course, or 3 = improved. The electrophysiology data were rated as $0=$ worse, $1=$ stable, or $2=$ improved. Statistical analyses were performed using JMP (version 9.0.2 for Macintosh, from SAS).
\end{abstract}

Results Seventy percent were males, aged $68.1 \pm 12.6$ years with neuropathy for $9.8 \pm 6.8$ years and follow-up of $4.0 \pm 3.2$ years. CIDP patients had more severe neuropathy, and were more likely to receive treatment and to respond. The clinical neuropathy status remained unchanged in $52.8 \%$ of the MGUSN and $24.2 \%$

N. M. Alkhawajah

Department of Medicine (Neurology), King Saud University, Riyadh, Saudi Arabia

N. M. Alkhawajah · S. K. Dunnigan · V. Bril

Department of Medicine (Neurology), University of Toronto,

Toronto, Canada

N. M. Alkhawajah · S. K. Dunnigan · V. Bril ( ()

Toronto General Hospital, Eaton Centre Wing, 5-309,

200 Elizabeth Street., Toronto, ON M5G 2C4, Canada

e-mail: vera.bril@utoronto.ca of the CIDP patients, and stabilized in $7.6 \%$ of MGUSN and $30.3 \%$ of CIDP patients. IgM-MGUSN patients did not differ from other immunoglobulin subclasses in response to treatment. The clinical severity and the number of abnormal NCS parameters were greater in the demyelinating MGUSN in comparison to the axonal group.

Conclusion MGUSN patients have less severe neuropathy than CIDP patients, but among the MGUSN patients the severity is greater in the demyelinating and the $\operatorname{IgM}$ groups. MGUSN patients may do well without treatment and exposure to potential adverse effects.

Keywords Monoclonal gammopathy of undetermined significance $\cdot$ Chronic inflammatory demyelinating polyneuropathy $\cdot$ Demyelinating neuropathy $\cdot$ Axonal neuropathy · Treatment

\section{Introduction}

Monoclonal gammopathies are a group of disorders characterized by excessive amounts of serum immunoglobulins produced by proliferation of a single clone of plasma cells [1]. Most of the patients have monoclonal gammopathy of undetermined significance (MGUS) and the rest have systemic diseases such as amyloidosis, multiple myeloma, osteosclerotic myeloma, lymphoma, Waldenstrom's macroglobulinemia, POEMS syndrome (polyneuropathy, organomegaly, endocrinopathy, monoclonal gammopathy and skin changes) and cryoglobulinemia [2-4]. Ten percent of patients with idiopathic neuropathy have monoclonal gammopathy, in contrast to $2.5 \%$ of patients with nonidiopathic neuropathy suggesting an adverse effect of the gammopathy on peripheral nerve function [5]. The most frequent type of immunoglobulin seen with MGUS is $\mathrm{IgG}$, 
and this is in contrast to MGUS-associated neuropathy (MGUSN) in which the most common type is IgM [3, 6]. MGUSN, classified into IgM and non-IgM associated [7], is an under diagnosed entity [8]. Chronic inflammatory demyelinating polyneuropathy (CIDP) is an acquired symmetrical progressive relapsing sensorimotor neuropathy, and it is reported that $22-30 \%$ of CIDP patients have MGUS [9-11]. There are varying reports on whether MGUSN patients are distinguishable from those with CIDP based on their clinical presentation, electrophysiologic findings and response to treatment [9-12].

We aimed to determine whether the clinical characteristics and response to treatment differed in MGUSN, or any subclass of MGUSN, and CIDP patients.

\section{Research design and methods}

\section{Subjects}

MGUSN and CIDP subjects attending the Neuromuscular Clinic at the University Health Network for management of their immune-mediated polyneuropathy between 1990 and 2013 were evaluated for this study. Our current study involved the extraction of demographic data, clinical history, physical examination, laboratory test results and electrophysiologic data from previously coded charts of MGUSN and CIDP patients. The Research Ethics Board at the University Health Network approved the current study protocol.

All subjects were $\geq 18$ years of age and had a diagnosis of CIDP or MGUSN. The 67 patients with CIDP comprised a cohort matched for age and gender to a CIDP plus diabetes patient cohort from a prior study [13]. The CIDP cohort was selected from a total of 1,900 patients with this diagnosis and was fully representative of the larger cohort. The MGUSN cohort included all patients diagnosed with this disorder in the study period. CIDP was diagnosed based on the clinical presentation, as judged by a neuromuscular expert $[\mathrm{VB}]$, and the presence of demyelination on nerve conduction studies (NCS). CIDP patients fulfilled the Koski criteria (chronic polyneuropathy progressive for at least 8 weeks, no serum paraprotein and no genetic abnormality and either recordable compound muscle action potentials in at least $75 \%$ of motor nerves and either abnormal distal latency or abnormal motor conduction velocity or abnormal $F$ wave latency in $>50 \%$ of motor nerves, OR, symmetric onset or symmetric examination findings and weakness in all four limbs and proximal weakness in at least one limb) [14]. Although these criteria were described later, the CIDP patients in this study fulfilled these criteria and were matched for age and gender to an existing cohort of diabetes patients with CIDP. MGUSN was diagnosed on identification of serum monoclonal proteins by immunoelectrophoresis after exclusion of plasma cell dyscrasias (multiple myeloma, osteosclerotic myeloma, POEM's syndrome, lymphoma, Waldenstrom's macroglobulinemia, amyloidosis) by a hematologist, and other possible etiologies of peripheral neuropathy with evaluation of FBS, HbA1c, 2-h GTT, CBC, ESR, anti-GM1 Ganglioside antibodies, LFTs, creatinine, vitamin B12, C3, $\mathrm{C} 4$, rheumatoid factor, anti-DS DNA, VDRL and in some cases Lyme serology, West Nile virus, CSF protein and cell count analysis.

\section{Evaluation}

All subjects were evaluated by neurological examination, the validated Toronto Clinical Neuropathy Score (TCNS) $[15,16]$, vibration perception threshold (VPT), and median; peroneal; tibial and sural NCS.

NCS were performed using the Sierra Wave Electromyography Instrument (Cadwell Laboratories Inc., Kennewick, WA, USA). Age- and height-adjusted NCS reference values were used, according to the standards of the Toronto General Hospital (TGH) University Health Network (UHN) electrophysiologic laboratory.

\section{Nerve conduction studies}

Median, peroneal, tibial and sural NCS were performed using surface stimulating and recording techniques according to the standards of the Canadian Society of Clinical Neurophysiologists and the American Association of Neuromuscular and Electrodiagnostic Medicine [17, 18]. The electromyography instrument measured distal latencies (DL) and amplitudes, and calculated conduction velocities (CV) automatically. Compound muscle action potential (CMAP) amplitude was measured as baseline to peak for the median, peroneal and tibial nerves. For the sural sensory nerve action potential (SNAP), the amplitude was measured as baseline to negative peak, or from the positive peak (if present) to the negative peak. The sural nerve latency was measured at onset from the initial deflection from the baseline. The $F$ wave latency was determined as the minimum reproducible latency obtained after 10 supra-maximal stimuli were applied.

At each subsequent visit, patients were assessed by history, clinical examination and repeat NCS. Change in polyneuropathy status was judged on both clinical and electrophysiologic grounds. Using the clinical data from the history and neurological examination at the last visit, the patients were rated as $0=$ worse, $1=$ unchanged, $2=$ stabilized after declining course, or $3=$ improved. Using the electrophysiology data, the patients were rated as follows: $0=$ worse, $1=$ stable or $2=$ improved. 
Statistical analyses

Statistical analyses were performed using JMP (version 9.0.2 for Macintosh, from SAS). Demographic data were expressed as mean \pm standard deviation (SD) for normally distributed data, or median and interquartile range (IQR) for non-parametric data. Differences in categorical variables were assessed using the $\chi^{2}$, while differences in continuous variables were assessed using the ANOVA. $p$ values $<0.05$ were considered significant.

\section{Results}

A total of 123 subjects with a mean age of $68.1 \pm 12.6$ years were entered into the study. The demographic profile of the patients is shown in Table 1 . About $70 \%$ of the patients were males in both groups. The mean duration of neuropathy was $9.8 \pm 6.8$ years and of follow-up was $4.0 \pm 3.2$ years. Neuropathy was more severe in those with CIDP as demonstrated by the findings of more abnormality of upper limb reflexes (score of 3 vs 0 , $p=0.005$ ), lower limb reflexes (score of 6 vs 4 , $p=0.009$ ) and also more generalized weakness on clinical examination with $28.8 \%$ of CIDP patients having generalized weakness compared with $5.6 \%$ of those with MGUSN $(p=0.002)$ although the severity of weakness was similar (Table 1). However, the percentage of those able to ambulate without aids did not differ between groups with about $75 \%$ of patients freely ambulatory. The greater severity of neuropathy in CIDP patients was evidenced by more abnormal nerve conduction parameters (median nerve CMAP amplitude and CV, peroneal nerve CV and tibial nerve $F$ wave latency) as shown in Table 2. This table shows only those NCS parameters that were significantly different, but all other NCS parameters tended to be worse in the CIDP group although not reaching a $p$ value of $<0.05$ (data not shown). Interestingly, lower limb VPT was more abnormal in the MGUSN group.

Ninety-two percent of the CIDP patients received treatment in comparison to $52 \%$ of the MGUSN patients $(p<0.0001)$. Eighty-six percent of the CIDP patients compared with $26.8 \%$ of MGUSN received intravenous immunoglobulin (IVIG), $65.7 \%$ CIDP patients versus $21.4 \%$ MGUSN prednisone, $53.7 \%$ CIDP patients versus $8.9 \%$ MGUSN azathioprine, $14.4 \%$ CIDP patients versus $17.9 \%$ MGUSN plasmapheresis, $13.4 \%$ CIDP patients versus $3.6 \%$ MGUSN mycophenolate mofetil, $3.0 \%$ CIDP patients versus $5.4 \%$ MGUSN rituximab, $3 \%$ CIDP patients versus $1.8 \%$ MGUSN cyclophosphamide, $1.5 \%$ CIDP patients versus 0 MGUSN methotrexate, and 0 CIDP versus $1.8 \%$ MGUSN chlorambucil. CIDP patients were more likely to receive IVIG, prednisone, and azathioprine $(p<0.0001)$, and were more likely to respond to IVIG $(p<0.0001)$. There was no difference in the response to other treatments between the MGUSN and CIDP patients. The clinical neuropathy status stabilized in $7.6 \%$ of MGUSN and $30.3 \%$ of CIDP ( $p=0.001)$, and remained unchanged in more than half of the MGUSN and in $24.2 \%$ of the CIDP patients $(p=0.001)$. There was no difference in the percentage of patients who improved or worsened in both groups. The NCS remained stable in the majority of MGUSN and CIDP patients.

In the MGUSN group, $46.4 \%$ had IgM, $39.3 \%$ had IgG, $9 \%$ had $\operatorname{IgA}, 3.6 \%$ had a combination, and in $1.8 \%$ the immunoglobulin type was unspecified. Forty-five percent of the MGUSN had kappa light chains. The patients were classified according to the heavy chain into two groups: IgM$\operatorname{MGUSN}(n=26)$ and non-IgM-MGUSN with IgG, IgA, or a combination $(n=30)$. The two groups were the same in age, gender, duration of neuropathy and the distribution of light chains (kappa or lambda). Anti-MAG antibodies were seen in $30.8 \%$ of IgM-MGUSN patients and in none of the non-IgMMGUSN patients $(p=0.001)$. IgM-MGUSN patients had more severe neuropathy as demonstrated by higher VPT values in both upper and lower limbs and a tendency to more abnormal NCS parameters, significantly so for the median and peroneal nerve distal motor latencies. There was no difference between the two groups in distribution of weakness. About $1 / 2$ the patients were treated in each group. There was no difference in the use of azathioprine, mycophenolate mofetil, rituximab, cyclophosphamide, methotrexate, IVIG or plasmapheresis between the two groups. Prednisone was used more commonly in non-IgM-MGUSN patients $(p=0.025)$. There was no difference in the response to any of the treatments. The clinical neuropathy status remained unchanged in about half of both groups. The NCS remained stable in about two-thirds of the patients in both groups. However, there was a trend towards more clinical and electrophysiologic improvement and less worsening in IgM-MGUSN patients. When comparing IgM-MGUSN patients to CIDP patients, there was no difference in severity (comparable TCNS and NCS with the exception of longer tibial nerve $F$ wave latencies in the CIDP group), but VPT was higher in those with IgM paraproteins. Comparing non-IgM-MGUSN to CIDP patients, CIDP patients had more severe neuropathy as demonstrated by both TCNS and NCS.

We stratified the MGUSN patients into demyelinating $(n=29)$ and axonal $(n=27)$ forms as shown in Table 3. We found that those classified as demyelinating had more lambda light chains (14 vs 5, $p=0.017$ ), had all the antiMAG antibody-positive patients ( 8 vs $0, p=0.005$ ) and had more severe polyneuropathy as shown by the VPT, TCNS data and the number of abnormal NCS parameters (Table 3). There was no difference in age, gender or duration of neuropathy. 
Table 1 Demographic profile of 123 patients with monoclonal gammopathy of undetermined significance-associated neuropathy (MGUSN) (56) and chronic inflammatory demyelinating polyneuropathy (CIDP) (67)

\begin{tabular}{|c|c|c|c|}
\hline Parameter & MGUSN & CIDP & $p$ value $^{\mathrm{a}}$ \\
\hline Age (years), mean $\pm S D$ & $70 \pm 11.5$ & $66.5 \pm 13.4$ & 0.124 \\
\hline Age range (years) & $(45-90)$ & $(36-90)$ & - \\
\hline Gender male (\%) & 70 & 72 & 0.808 \\
\hline $\begin{array}{l}\text { Duration of follow-up (years), } \\
\text { mean } \pm \text { SD }\end{array}$ & $4.5 \pm 0.39$ & $3.3 \pm 0.44$ & 0.037 \\
\hline $\begin{array}{l}\text { Duration of peripheral } \\
\text { neuropathy (years), } \\
\text { mean } \pm S D\end{array}$ & $9.9 \pm 6.1$ & $9.7 \pm 7.4$ & 0.920 \\
\hline $\begin{array}{l}\text { TCNS_symptoms }{ }^{\mathrm{b}} \text {, Median } \\
\text { (IQR) }\end{array}$ & $3(2-4)$ & $4(3-5)$ & 0.181 \\
\hline $\begin{array}{l}\text { TCNS_sensory }{ }^{\mathrm{c}} \text {, Median } \\
\text { (IQR) }\end{array}$ & $4(2-5)$ & $4(2-4)$ & 0.496 \\
\hline $\begin{array}{l}\text { TCNS_deep tendon reflexes } \\
\text { Median (IQR) }\end{array}$ & $4(1.25-6)$ & $6(4-8)$ & 0.009 \\
\hline TCNS_totale, Median (IQR) & $11(7-14)$ & $13(8-16)$ & 0.053 \\
\hline Weakness on exam ${ }^{\mathrm{f}}, n(\%)$ & $22(39.3)$ & $34(50.8)$ & 0.203 \\
\hline Weakness proximal ${ }^{\mathrm{f}}, n(\%)$ & $3(5.4)$ & $3(4.5)$ & 1 \\
\hline Weakness distal $^{\mathrm{f}}, n(\%)$ & $14(25)$ & $16(23.9)$ & 0.886 \\
\hline Weakness generalized $^{\mathrm{f}}, n(\%)$ & $3(5.6)$ & $19(28.8)$ & 0.002 \\
\hline Gait abnormal, $n(\%)$ & $30(53.6)$ & $34(50.8)$ & 0.755 \\
\hline Independent walking (\%) & 78.6 & 74.6 & 0.61 \\
\hline Treated patients, $n(\%)$ & $29(51.8)$ & $62(92.5)$ & $<0.0001$ \\
\hline IVIG, $n(\%)$ & $15(26.8)$ & $58(86.6)$ & $<0.0001$ \\
\hline Prednisone, $n(\%)$ & $12(21.4)$ & $44(65.7)$ & $<0.0001$ \\
\hline Plasmapheresis, $n(\%)$ & $10(17.9)$ & $10(14.4)$ & 0.661 \\
\hline Azathioprine, $n(\%)$ & $5(8.9)$ & $36(53.7)$ & $<0.0001$ \\
\hline Mycophenolate mofetil, $n(\%)$ & $2(3.6)$ & $9(13.4)$ & 0.065 \\
\hline Rituximab, $n(\%)$ & $3(5.4)$ & $2(3)$ & 0.659 \\
\hline Cyclophosphamide, $n(\%)$ & $1(1.8)$ & $2(3.0)$ & 1 \\
\hline Methotrexate, $n(\%)$ & $0(0)$ & $1(1.5)$ & 1 \\
\hline Chlorambucil, $n(\%)$ & $1(1.8)$ & $0(0)$ & 0.459 \\
\hline
\end{tabular}

$I V I G$ intravenous immunoglobulin

${ }^{a} p$ values $<0.05$ are considered significant

b Toronto clinical neuropathy score_symptoms: present $=1$; absent $=0(0-6)$

c Toronto clinical neuropathy score_sensory: abnormal $=1$; normal $=0(0-5)$

d Toronto clinical neuropathy score_deep tendon reflexes: absent $=2$; reduced $=1$; normal $=0(0-8)$

e Toronto clinical neuropathy score_total: normal $=0$ to maximum of 19

${ }^{\mathrm{f}}$ Weakness determined by Medical Research Council (MRC) grading of muscles

\section{Discussion}

We examined our cohort of MGUSN patients and compared them to an existing cohort of CIDP patients to
Table 2 Quantitative sensory threshold and nerve conduction testing in 123 patients with monoclonal gammopathy of undetermined significance-associated neuropathy (MGUSN) (56) and chronic inflammatory demyelinating polyneuropathy (CIDP) (67)

\begin{tabular}{|c|c|c|c|}
\hline Parameter & MGUSN $^{\mathrm{a}}$ & $\mathrm{CIDP}^{\mathrm{a}}$ & $p$ value $^{\mathrm{b}}$ \\
\hline VPT-right upper limb & $\begin{array}{r}6.20 \pm 3.54 \\
(1.7-23.3)\end{array}$ & $\begin{array}{l}6.32 \pm 5.28 \\
(2-39)\end{array}$ & 0.894 \\
\hline VPT-left upper limb & $\begin{array}{r}6.13 \pm 3.56 \\
(1.8-21.8)\end{array}$ & $\begin{array}{l}6.23 \pm 5.33 \\
(2-39.7)\end{array}$ & 0.912 \\
\hline VPT-right lower limb & $\begin{array}{l}31.33 \pm 15.75 \\
\quad(4-50)\end{array}$ & $\begin{array}{l}24.07 \pm 13.87 \\
(3.2-50)\end{array}$ & 0.011 \\
\hline VPT-left lower limb & $\begin{array}{l}30.41 \pm 15.6 \\
\quad(4.2-50)\end{array}$ & $\begin{array}{l}23.9 \pm 13.6 \\
(3.2-50)\end{array}$ & 0.020 \\
\hline $\begin{array}{l}\text { Median CMAP Wrist, } \\
\mathrm{mV}\end{array}$ & $\begin{array}{r}9.7 \pm 5.3 \\
(0-30.5)\end{array}$ & $\begin{array}{l}7.45 \pm 4.4 \\
(0-19.9)\end{array}$ & 0.012 \\
\hline $\begin{array}{l}\text { Median CMAP } \\
\text { Elbow, mV }\end{array}$ & $\begin{array}{l}8.8 \pm 5.3 \\
(0-28.7)\end{array}$ & $\begin{array}{l}6.54 \pm 4.2 \\
(0-19.4)\end{array}$ & 0.011 \\
\hline Median CV, m/s & $\begin{array}{l}46.9 \pm 9.9 \\
(12-63)\end{array}$ & $\begin{array}{l}42.1 \pm 10.6 \\
(8-60.4)\end{array}$ & 0.013 \\
\hline $\begin{array}{l}\text { Peroneal CV Fibular } \\
\text { head, } \mathrm{m} / \mathrm{s}\end{array}$ & $\begin{array}{c}37 \pm 8.8 \\
(7-51)\end{array}$ & $\begin{array}{l}33.7 \pm 7.4 \\
(15-47)\end{array}$ & 0.049 \\
\hline $\begin{array}{l}\text { Peroneal CV Popliteal } \\
\text { fossa, } \mathrm{m} / \mathrm{s}\end{array}$ & $\begin{array}{r}39.4 \pm 9 \\
(11-55)\end{array}$ & $\begin{array}{l}35.41 \pm 7.70 \\
(15-53)\end{array}$ & 0.021 \\
\hline $\begin{array}{l}\text { Tibial } F \text { wave latency, } \\
\text { ms }\end{array}$ & $\begin{array}{l}60.9 \pm 7.6 \\
(45.6-77.2)\end{array}$ & $\begin{array}{l}66.6 \pm 6.7 \\
\quad(53.8-86.3)\end{array}$ & 0.008 \\
\hline
\end{tabular}

$V P T$ vibration perception threshold, CMAP compound muscle action potential amplitude, $C V$ conduction velocity

${ }^{a}$ Values are shown as means \pm standard deviations (range)

${ }^{\mathrm{b}} p$ values $<0.05$ are considered significant

determine whether the clinical characteristics and response to treatment differed between the groups or in subclasses of MGUSN.

The age, gender and the duration of the polyneuropathy did not differ between the two groups in this study, similar to findings in a previous report [10]. However, other authors found that CIDP-MGUS patients were more likely to be older males in comparison to idiopathic CIDP (CIDPI) $[11,12]$.

In our cohort, CIDP patients had more severe neuropathy based on both clinical and electrodiagnostic measures. These differences were most apparent between non-IGMMGUSN and CIDP patients (data not shown) than between IgM-MGUSN and CIDP patients. Several studies compared CIDP patients with and without MGUS [9-12], but none-up to our knowledge-compared purely axonal MGUSN to CIDP or subclasses of MGUSN to CIDP. Our results are similar to those of Simmons et al. [9] who found less severe weakness and a more indolent course in CIDPMGUS. However, they contrast with Gorson et al. [10] who found that CIDP-MGUS patients did not have a more indolent course despite the fact that weakness was less common in this group, and Bromberg et al. who found that CIDP-MGUS cannot be distinguished from CIDP-I on the 
Table 3 Patients with monoclonal gammopathy of undetermined significance-associated neuropathy (MGUSN) classified as demyelinating or axonal polyneuropathy

\begin{tabular}{|c|c|c|c|}
\hline Parameter & Demyelinating & Axonal & $p$ value $^{\mathrm{a}}$ \\
\hline Gender male (\%) & 69 & 70.4 & 0.91 \\
\hline Age (years), mean $\pm \mathrm{SD}$ & $70 \pm 11.4$ & $70 \pm 11.7$ & 0.982 \\
\hline $\begin{array}{l}\text { Duration of follow-up in } \\
\text { years, mean } \pm S D\end{array}$ & $3.2 \pm 2.7$ & $3.3 \pm 3.5$ & 0.913 \\
\hline $\mathrm{IgG}, n(\%)$ & $9(31)$ & $13(48.2)$ & 0.189 \\
\hline $\operatorname{IgM}, n(\%)$ & $17(58.6)$ & $9(33.3)$ & 0.057 \\
\hline $\operatorname{IgA}, n(\%)$ & $2(6.9)$ & $3(11.1)$ & 0.58 \\
\hline $\operatorname{IgM}$ and $\operatorname{IgG}, n(\%)$ & 0 & $1(3.7)$ & 0.482 \\
\hline $\operatorname{IgG}$ and $\operatorname{IgA}, n(\%)$ & 0 & $1(3.7)$ & 0.482 \\
\hline Unspecified, $n(\%)$ & $1(3.5)$ & 0 & 1 \\
\hline Lambda, $n(\%)$ & $14(48.3)$ & $5(18.5)$ & 0.017 \\
\hline Kappa, $n(\%)$ & $10(34.5)$ & $15(55.6)$ & 0.112 \\
\hline Lambda and kappa, $n(\%)$ & $1(3.5)$ & $5(18.5)$ & 0.096 \\
\hline Unspecified, $n(\%)$ & $4(13.8)$ & $2(7.4)$ & 0.671 \\
\hline Anti-MAG, $n(\%)$ & $8(27.6)$ & 0 & 0.005 \\
\hline $\begin{array}{l}\text { VPT-right upper limb, } \\
\text { mean } \pm \text { SD }\end{array}$ & $7.2 \pm 4.5$ & $5.1 \pm 1.7$ & 0.343 \\
\hline $\begin{array}{l}\text { VPT-left upper limb, } \\
\text { mean } \pm \text { SD }\end{array}$ & $7.2 \pm 4.5$ & $5.0 \pm 1.7$ & 0.029 \\
\hline $\begin{array}{l}\text { VPT-right lower limb, } \\
\text { mean } \pm \text { SD }\end{array}$ & $38.4 \pm 14.6$ & $24.0 \pm 13.7$ & 0.001 \\
\hline $\begin{array}{l}\text { VPT-left lower limb, } \\
\text { mean } \pm \text { SD }\end{array}$ & $37.4 \pm 14.5$ & $23.9 \pm 14.0$ & 0.001 \\
\hline $\begin{array}{l}\text { TCNS-symptoms } \\
\text { mean } \pm \text { SD }\end{array}$ & $3.7 \pm 1.4$ & $3.0 \pm 1.3$ & 0.059 \\
\hline $\begin{array}{l}\text { TCNS-sensory }^{\mathrm{c}} \\
\text { mean } \pm \mathrm{SD}\end{array}$ & $3.8 \pm 1.3$ & $2.8 \pm 1.9$ & 0.026 \\
\hline $\begin{array}{l}\text { TCNS-deep tendon } \\
\text { reflexes }^{\mathrm{d}} \text {, mean } \pm \mathrm{SD}\end{array}$ & $4.7 \pm 2.8$ & $3.1 \pm 2.6$ & 0.037 \\
\hline TCNS-total $^{\mathrm{e}}$, mean \pm SD & $12.1 \pm 3.8$ & $8.9 \pm 4.1$ & 0.004 \\
\hline $\begin{array}{l}\text { Number of abnormal nerve } \\
\text { conduction studies } \\
\text { parameters }\end{array}$ & $8.1 \pm 2.5$ & $5.7 \pm 3.5$ & 0.005 \\
\hline
\end{tabular}

$M A G$ myelin associated glycoprotein, $V P T$ vibration perception threshold

${ }^{\text {a }} p$ values $<0.05$ are considered significant

b Toronto clinical neuropathy score_symptoms: present $=1$; absent $=0(0-6)$

${ }^{c}$ Toronto clinical neuropathy score_sensory: abnormal $=1$; normal $=0(0-5)$

d Toronto clinical neuropathy score_deep tendon reflexes: absent $=2$; reduced $=1$; normal $=0(0-8)$

e Toronto clinical neuropathy score_total: normal $=0$ to maximum of 19

basis of NCS [11]. In our cohort, we found differences but particular cut-off values for individual patients would require a different study. About $90 \%$ of the CIDP patients in comparison to $50 \%$ of the MGUSN patients in our cohort were treated, likely due to greater severity of the disease in the CIDP patients warranting treatment. More CIDP patients were stabilized compared to MGUSN patients, but there were no differences in patients categorized as improving or worsening. Because of the uneven number of treated patients in both groups, we examined the outcome of the treated MGUSN patients only and compared it to the treated CIDP patients. We found the post treatment clinical and electrophysiologic status to be the same in both groups, except that more treated CIDP patients stabilized in comparison to treated MGUSN patients (32.8 versus $10.3 \%$, respectively, $p=0.037$ ). The CIDP patients had more responders to IVIG treatment when compared to all MGUSN patients combined or when compared to those with IgM- or non-IGM-MGUSN patients, and had more responders to plasmapheresis when compared to non-IgM-MGUSN patients in contrast to an earlier report [10]. In a long-term follow-up by Simmons et al. [12] in which $90 \%$ of the CIDP-I and $80 \%$ of the CIDP-MGUS patients were treated, CIDP-MGUS had more functional impairment at the end of the follow-up period, but we do not find a similar outcome in this study.

It is thought that MGUSN patients have demyelinating disease and that axonal features are secondary [19], but Notermans et al. [20] found that $40 \%$ of MGUSN are purely axonal. Filostso et al. [8] detected pure axonal findings in $46 \%$ of $\operatorname{IgG}$ and $20 \%$ of IgM-MGUSN patients. In our cohort, about $48 \%$ of the MGUSN patients had primarily axonal neuropathy and $52 \%$ were primarily demyelinating. The lambda paraprotein was observed more frequently in the demyelinating category and all anti-MAG antibody-positive patients fell into this group, although the prevalence of anti-MAG was less than described by Filosto et al. [8]. Although there were no differences in the gender, age of onset or duration of the peripheral neuropathy, the clinical severity and the number of abnormal NCS parameters were greater in the demyelinating group as was the percentage of treated patients $(70 \%$ of the demyelinating vs $30 \%$ of the axonal, $p=0.001)$. In our cohort, axonal and demyelinating MGUSN patients responded similarly to treatment in contrast to the experience of Gorson et al. [19].

As reported previously [21-23], there were no differences between subclasses of MGUSN (IgM versus others) with respect to duration of peripheral neuropathy, age, gender or presenting symptoms, or signs. However, others found that IgM-MGUSN patients are more likely to have sensory dysfunction, progressive course, and a more severe predominantly demyelinating polyneuropathy in comparison to other subclasses [20-22, 24, 25]. On NCS we found that IgM-MGUSN group had longer distal motor latencies in the upper and lower limbs similar to the findings of Suarez and Kelly [24]. There was no difference in response to treatment between the two groups as reported previously 
by Simovic et al. [21], this is in contrast to Yeung et al. [23] who concluded that IgG- and IgA-MGUSN patients are more likely to respond to treatment than IgM-MGUSN patients.

Our results support the concept that CIDP patients differ from those with MGUSN. The MGUSN clinical phenotype shows milder disease that leads less frequently to treatment although IgM-MGUSN patients had more severe disease within the MGUSN group. Further, demyelinating MGUSN patients had more severe clinical disease with significantly more abnormal NCS parameters in comparison to axonal MGUSN. As well, treatment response rate appears to be lower in MGUSN patients, adding some validity to the choice to defer treatment in these patients. Given the potential adverse effects of most immunosuppressive treatments, and the expense and limited access to immunomodulation treatments, it is important to determine the best therapeutic choices for patients with immunemediated polyneuropathies. Our study suggests that those with MGUSN may do well without treatment in many cases and patients can avoid exposure to adverse effects and high costs. Even with treatment, it should be noted that only few patients stabilized or improved.

The limitations of our study are that it is a retrospective review with inherent difficulties in standardized evaluations, treatments, and data collection; however, all reports comparing MGUSN to CIDP - up to our knowledge - have been retrospective to date [9-12,26]. Also, the CIDP cohort was selected for a prior study for age and gender factors, and not all patients diagnosed with CIDP in our clinic within the timeframe of the study were included and thus the results may not be generalizable to all CIDP patients. Some novel and improved measures in inflammatory neuropathies such as the Rasch overall disability scale [27] and the overall neuropathy limitation scale (ONLS) [28] were not available when these patients were evaluated and their use might have provided better insight into patient outcomes. In addition, the TCNS is not a linear scale and sensitivity to change may be limited. Finally, MGUSN patients had milder disease with a floor effect for improvement. Further, although we defined the demyelinating categories on the basis of NCS, we do not have morphological confirmation of the underlying pathology. Despite these considerations, our study suggests that MGUSN differs from CIDP both in clinical severity and response to treatment. MGUSN is a heterogenous disorder and alone it shows variability between IgM and nonIgM forms. A multi-center prospective natural history and intervention study might allow better determination of which MGUSN patients to treat.

Conflicts of interest On behalf of all authors, the corresponding author states that there is no conflict of interest.

Ethical standard This study was carried in accordance with ethical standards as set out in the Declaration of Helsinki.
Open Access This article is distributed under the terms of the Creative Commons Attribution License which permits any use, distribution, and reproduction in any medium, provided the original author(s) and the source are credited.

\section{References}

1. Kyle RA (1978) Monoclonal gammopathy of undetermined significance natural history in 241 cases. Am J Med 64:814-826

2. Kissel JT, Mendell JR (1996) Neuropathies associated with monoclonal gammopathies. Neuromuscul Disord 6(1):3-18

3. Ramchandren S, Lewis RA (2009) Monoclonal gammopathy and neuropathy. Curr Opin Neurol 22(5):480-485. doi:10.1097/ WCO.0b013e32832fd563

4. Kelly JJ Jr (1985) Peripheral neuropathies associated with monoclonal proteins: a clinical review. Muscle Nerve 8(2): 138-150. doi:10.1002/mus.880080210

5. Kelly JJ Jr, Kyle RA, O'Brien PC, Dyck PJ (1981) Prevalence of monoclonal protein in peripheral neuropathy. Neurology 31(11): $1480-1483$

6. Ameis A, Ko HS, Pruzanski W (1976) M components-a review of 1242 cases. Can Med Assoc J 114 (10):889-892, 895

7. Kwan JY (2007) Paraproteinemic neuropathy. Neurol Clin 25(1):47-69. doi:10.1016/j.ncl.2006.12.002

8. Filosto M, Cotelli M, Todeschini A, Broglio L, Vielmi V, Rinaldi F, Gregorelli V, Benelle M, Padovani A (2012) Clinical spectrum and evolution of monoclonal gammopathy-associated neuropathy: an observational study. Neurologist 18(6):378-384. doi:10. 1097/NRL.0b013e31826a99e9

9. Simmons Z, Albers JW, Bromberg MB, Feldman EL (1993) Presentation and initial clinical course in patients with chronic inflammatory demyelinating polyradiculoneuropathy: comparison of patients without and with monoclonal gammopathy. Neurology 43(11):2202-2209

10. Gorson KC, Allam G, Ropper AH (1997) Chronic inflammatory demyelinating polyneuropathy: clinical features and response to treatment in 67 consecutive patients with and without a monoclonal gammopathy. Neurology 48(2):321-328

11. Bromberg MB, Feldman EL, Albers JW (1992) Chronic inflammatory demyelinating polyradiculoneuropathy: comparison of patients with and without an associated monoclonal gammopathy. Neurology 42(6):1157-1163

12. Simmons Z, Albers JW, Bromberg MB, Feldman EL (1995) Long-term follow-up of patients with chronic inflammatory demyelinating polyradiculoneuropathy, without and with monoclonal gammopathy. Brain 118(Pt 2):359-368

13. Dunnigan SK, Ebadi H, Breiner A, Katzberg HD, Barnett C, Perkins BA, Bril V (2014) The characteristics of chronic inflammatory demyelinating polyneuropathy in patients with and without diabetes-an observational study. PLoS One 9(2):e89344. doi:10.1371/journal.pone.0089344

14. Koski CL, Baumgarten M, Magder LS, Barohn RJ, Goldstein J, Graves M, Gorson K, Hahn AF, Hughes RA, Katz J, Lewis RA, Parry GJ, van Doorn P, Cornblath DR (2009) Derivation and validation of diagnostic criteria for chronic inflammatory demyelinating polyneuropathy. J Neurol Sci 277(1-2):1-8. doi:10. 1016/j.jns.2008.11.015

15. Bril V, Tomioka S, Buchanan RA, Perkins BA (2009) Reliability and validity of the modified Toronto Clinical Neuropathy Score in diabetic sensorimotor polyneuropathy. Diabet Med 26(3): 240-246. doi:10.1111/j.1464-5491.2009.02667.x

16. Bril V, Perkins BA (2002) Validation of the Toronto Clinical Scoring System for diabetic polyneuropathy. Diabetes Care 25(11):2048-2052 
17. Bolton CF, Benstead TJ, Grand'Maison F, Tardif GS, Weston LE (2000) Minimum standards for electromyography in Canada: a statement of the Canadian Society of Clinical Neurophysiologists. Can J Neurol Sci 27(4):288-291

18. AANEM (2004) Recommended policy for electrodiagnostic medicine. American Association of Neuromuscular and Electrodiagnostic Medicine. 1:1-16

19. Gorson KC, Ropper AH (1997) Axonal neuropathy associated with monoclonal gammopathy of undetermined significance. J Neurol Neurosurg Psychiatry 63(2):163-168

20. Notermans NC, Wokke JH, Lokhorst HM, Franssen H, van der Graaf Y, Jennekens FG (1994) Polyneuropathy associated with monoclonal gammopathy of undetermined significance. A prospective study of the prognostic value of clinical and laboratory abnormalities. Brain 117(Pt 6):1385-1393

21. Simovic D, Gorson KC, Ropper AH (1998) Comparison of IgMMGUS and IgG-MGUS polyneuropathy. Acta Neurol Scand 97(3):194-200

22. Gosselin S, Kyle RA, Dyck PJ (1991) Neuropathy associated with monoclonal gammopathies of undetermined significance. Ann Neurol 30(1):54-61. doi:10.1002/ana.410300111

23. Yeung KB, Thomas PK, King RH, Waddy H, Will RG, Hughes RA, Gregson NA, Leibowitz S (1991) The clinical spectrum of peripheral neuropathies associated with benign monoclonal IgM,
IgG and $\operatorname{IgA}$ paraproteinaemia. Comparative clinical, immunological and nerve biopsy findings. J Neurol 238(7):383-391

24. Suarez GA, Kelly JJ Jr (1993) Polyneuropathy associated with monoclonal gammopathy of undetermined significance: further evidence that IgM-MGUS neuropathies are different than IgGMGUS. Neurology 43(7):1304-1308

25. Vrethem M, Reiser N, Lauermann C, Svanborg E (2010) Polyneuropathy associated with IgM vs IgG monoclonal gammopathy: comparison between clinical and electrophysiological findings. Acta Neurol Scand 122(1):52-57. doi:10.1111/j.16000404.2009.01259.x

26. Maisonobe T, Chassande B, Verin M, Jouni M, Leger JM, Bouche P (1996) Chronic dysimmune demyelinating polyneuropathy: a clinical and electrophysiological study of 93 patients. J Neurol Neurosurg Psychiatry 61(1):36-42

27. van Nes SI, Vanhoutte EK, van Doorn PA, Hermans M, Bakkers M, Kuitwaard K, Faber CG, Merkies IS (2011) Rasch-built Overall Disability Scale (R-ODS) for immune-mediated peripheral neuropathies. Neurology 76(4):337-345. doi:10.1212/WNL. 0b013e 318208824b

28. Graham RC, Hughes RA (2006) A modified peripheral neuropathy scale: the Overall Neuropathy Limitations Scale. J Neurol Neurosurg Psychiatry 77(8):973-976. doi:10.1136/jnnp.2005. 081547 